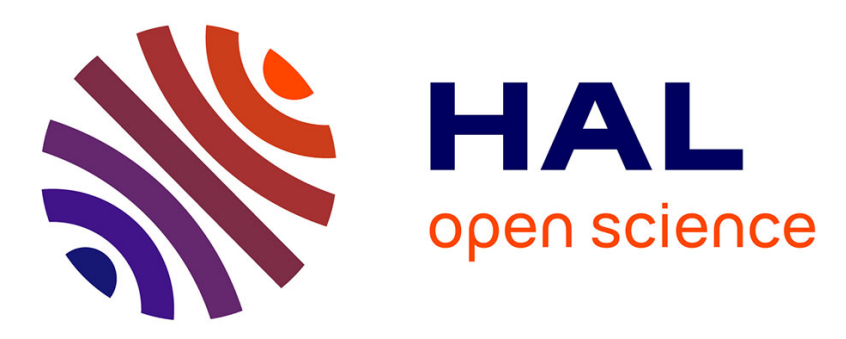

\title{
Legible robot navigation in the proximity of moving humans
}

\author{
Thibault Kruse, Patrizia Basili, Stefan Glasauer, Alexandra Kirsch
}

\section{To cite this version:}

Thibault Kruse, Patrizia Basili, Stefan Glasauer, Alexandra Kirsch. Legible robot navigation in the proximity of moving humans. IEEE workshop on Advanced Robotics and its Social Impacts, May 2012, Munich, Germany. pp.83 - 88, 10.1109/ARSO.2012.6213404 . hal-01571940

\author{
HAL Id: hal-01571940 \\ https://hal.science/hal-01571940
}

Submitted on 7 Aug 2017

HAL is a multi-disciplinary open access archive for the deposit and dissemination of scientific research documents, whether they are published or not. The documents may come from teaching and research institutions in France or abroad, or from public or private research centers.
L'archive ouverte pluridisciplinaire HAL, est destinée au dépôt et à la diffusion de documents scientifiques de niveau recherche, publiés ou non, émanant des établissements d'enseignement et de recherche français ou étrangers, des laboratoires publics ou privés. 


\title{
Legible Robot Navigation in the Proximity of Moving Humans
}

\author{
Thibault Kruse*, Patrizia Basili ${ }^{\dagger}$, Stefan Glasauer $^{\dagger}$ and Alexandra Kirsch* \\ ${ }^{*}$ Technische Universität München, Department of Informatics \\ ${ }^{\dagger}$ Ludwig-Maximilians-Universität München, Institute of Clinical Neurosciences
}

\begin{abstract}
Our objective is to improve legibility of robot navigation behavior in the presence of moving humans. We examine a human-aware global navigation planner in a path crossing situation and assess the legibility of the resulting navigation behavior. We observe planning based on fixed social costs and static search spaces to perform badly in situations where robot and human move towards the same point. To find an improved cost model, we experimentally examine how humans deal with path crossing. Based on the results we provide a new way of calculating social costs with context dependent costs without increasing the search space. Our evaluation shows that a simulated robot using our new cost model moves more similar to humans. This shows how comparison of human and robot behavior can help with assessing and improving legibility.
\end{abstract}

\section{INTRODUCTION}

Beyond guaranteeing safety, an autonomous robot needs to be acceptable as a partner in joint tasks. Social competencies are therefore necessary in robot systems when interacting with a human partner in order to achieve confidence, comfort and social acceptance from the human side [1]. For social competencies we considered in this work especially the robot's ability to behave in such a way that a human partner does not feel aggravated or afraid by the robot's movements and can easily infer the intentions of the robot. As a framework scenario we assume that the wider context of the robot navigation is to perform joint household activities with a human, such as setting a table or helping with the dishwasher.

Moving ahead from research on static situations, we focus on a robot navigation in the proximity of moving humans ensuring maximum comfort and legibility in dynamic situations. Legibility means that a person intuitively understands the intentions of a robot. We specifically consider situations with a small number of moving agents as they can occur in household and service environments. In such situations the position of the human and the robot as well as the goals of both agents change frequently and often unpredictably.

Socially acceptable behavior can to some extent be modeled by an explicit cost function. The Human-Aware Navigation Planner (HANP)[2] ${ }^{1}$, which we use as a global planner in this work, includes social constraints as cost models for robot navigation to ensure personal comfort. In this way, the robot plans paths that, for instance, avoid areas outside the human field of view and keep the robot from moving closely behind the back of the person.

In this paper we show that planning using static social costs in the form of a potential field can lead to illegible

\footnotetext{
${ }^{1}$ Original name HAMP changed to HANP in [3]
}

robot behavior. We develop a novel strategy which breaks with potential field assumptions and produces behavior that is similar to that observed in humans in experiments.

Our goal in this work is not to find a psychological model for human behavior, but to implement robot navigation in a way that results in a similar behavior to humans. This means that humans might have very different strategies for navigation than our robot, but the demonstrated behavior should be alike. We are also not interested in the movements of the limbs. We use a wheeled robot with a differential drive and focus on the navigation paths and speed.

In the following sections we first introduce HANP and its relation to other navigation planning approaches. After that we describe the experiment on human navigation behavior. We point out related work on navigation and on psychological studies of human locomotion in the respective sections. Then we explain our new cost model and evaluate it against the observed navigation behavior of humans.

\section{NAVigation PlanNing}

Navigation planning builds a plan to move from a current pose $\left(x_{0}, y_{0}, \theta_{0}\right)$ to a goal pose $\left(x_{g}, y_{g}, \theta_{g}\right)$. A general overview of classical motion planning techniques is given in [4].

A standard way to achieve both reactive behavior and global correctness is a separation of navigation frameworks into global and local planner. The local planner updates the velocity command with a high frequency towards a shortterm goal that may not be the global goal.

A global planning process updates in less frequent intervals the short-term goal as direction in which the local planner should move next. As a simple example, a robot in a dynamic maze may have a global planner planning the global route repeatedly, while the local planner only considers the next corner of the maze to move to. Local planners for dynamic worlds are not in the focus of this paper but subject to ongoing research [5], [6].

Global planners typically represent the space as a set of discrete grid cells, which are build from sensor data, identifying "allowed" grid cells into which the robot footprint fits as opposed to "blocked" cells where the robot cannot be, near walls and furniture. Given such a grid, a global planner returns a sequence of waypoints to follow, one neighboring the next in the grid (see Figure 1c), such that if the robot moves through the waypoints in sequence, it will reach the goal position without collisions. 


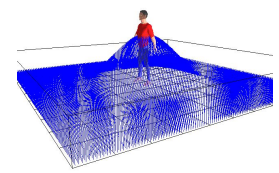

(a) Safety

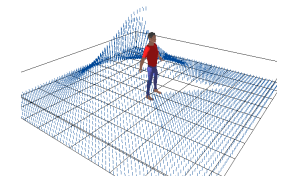

(b) Visibility

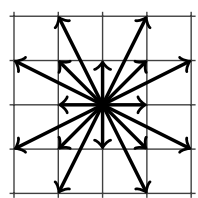

(c)
Fig. 1: (a), (b) Examples for social cost functions, (c) Neighboring cells considered in grid-based path planning.

\section{A. Human Awareness}

In human aware navigation, humans are treated differently from other obstacles. As an example in Hansen et al. [7], a robot approaches humans differently based on the human's activity, which is categorized by motion recognition. Gockley et al. [8] found out that a robot following a human is perceived as more comfortable when matching direction rather than matching path. These existing approaches all show how navigation for robots can take humans into account differently from generalized moving obstacles.

The human-aware navigation planner HANP that we use in this paper defines cost functions representing human discomfort based on the concept of proxemics, which was introduced by the anthropologist Edward T. Hall [9]. It is the most common attempt in robotics to describe how closely agents should be approached in different contexts to avoid discomfort. Based on his work, conceptual regions or "bubbles" around humans can intersect and cause reactions such as discomfort. The same has been shown for animals and robots [10], [11].

HANP finds robot paths in 2-dimensional square grids. The grid represents a map of the world with obstacles and humans. The paths are found using the $\mathrm{A}^{*}$ algorithm and they have the property of having a minimal weighted sum of distance and accumulated social costs.

For a single grid cell $w_{i}$ and each human $H=$ $\left(x_{H}, y_{H}, \theta_{H}, v_{H}\right)$ represented as $2 \mathrm{~d}$ pose $\left(x_{H}, y_{H}, \theta_{H}\right)$ with velocity $v_{H}$, we define a comfort cost function $\varsigma_{\text {Static }}$. Equation 1 shows how such a cost function can be based on several different specialized functions $f_{j}$, such as for visibility or safety as depicted in Figure 1.

$$
\varsigma_{\text {Static }}\left(H, w_{i}\right)=\max \left(f_{1}\left(H, w_{i}\right), f_{2}\left(H, w_{i}\right) \ldots, f_{k}\left(H, w_{i}\right)\right)
$$

Following the cost function given in HANP [2, page 31] we define the comfort costs $\sigma$ for a set of $n$ present humans $\mathscr{H}=\left\{H_{1}, \ldots H_{n}\right\}$ as follows:

$$
\sigma\left(\mathscr{H}, w_{i}\right)=\max _{H_{j} \in \mathscr{H}}\left(\varsigma_{\text {Static }}\left(H_{j}, w_{i}\right)\right)
$$

Definition 1: The costs of a path $P$ in the presence of humans $\mathscr{H}$ of length $l$ are a weighted sum of distance costs and social costs with weights $\omega_{\delta}$ and $\omega_{\sigma}$.

$$
\gamma(\mathscr{H}, P)=\sum_{i=2}^{l}\left(\omega_{\delta} \delta\left(w_{i-1}, w_{i}\right)+\omega_{\sigma} \sigma\left(\mathscr{H}, w_{i}\right)\right)
$$

HANP uses the Euclidean distance as distance measure $\delta$. The value of $\omega_{\delta}$ and $\omega_{\sigma}$ depends on the ranges of the functions $f_{j}$ and $\delta$, and there is currently no formal way to determine optimal values. We used a ratio of $\omega_{\delta} / \omega_{\sigma}=10$. The path with minimal costs is assumed to be the most human-aware, acceptable path.

\section{B. Legible Navigation Behavior}

With legibility we mean behavior that is intuitively understood by humans. In navigation specifically this refers to the motions of the robot indicating a goal direction (i.e. the goal pose the robot is trying to reach) and the robot visibly acknowledging the presence and status of obstacles and humans (i.e. taking into account the human presence and motion). Legibility also implies that the robot should react quickly and appropriately to new events.

Kruse et al. [12], [13] have proposed different strategies for plan execution to make the navigation with HANP more legible. The focus of that work was navigation in confined areas and at that time there was no comparative data of human behavior available, so that the results could only be judged from an intuitive point of view.

\section{Static Cost MOdel}

All global planning methods implicitly or explicitly make assumptions about the future via their cost model. If the future turns out differently than assumed by the cost model, a path that was considered optimal may not be useful anymore. The default approach is to assume in the costs that the world will not change and to replan whenever the world state changes.

Per default HANP uses a static cost model as described in Section II-A. To evaluate the legibility of this strategy, we chose a particularly challenging case, in which the intended robot path crosses that of a moving human in a right angle. We used the physical Gazebo simulator as an evaluation environment, in which one robot with a fixed strategy (called the "interferer" as in the human-human experiment described in section IV) represents the human movement and a second robot navigates autonomously to its goal pose using HANP with the replanning strategy (see Figure 4a). The robot we used for the intereferer as well as the autonomous robot is a B21 with a differential wheel drive. The coordinates in the experiment were chosen to match the human-human experiment described in section IV.

HANP was used as the global planner with the costs defined in [2] with a grid width $\varepsilon=15 \mathrm{~cm}$. As neighbor predicate $N$ for HANP paths we allowed all pairs of cells as shown in Figure 1c.

Figure 2a shows a representative path taken by a robot in the simulation. We plotted $(x, y)$ positions of the interferer robot and the autonomous robot in regular time intervals. Figure $2 \mathrm{~b}$ shows the average behavior over 100 trials. The variance in Figure $2 \mathrm{~b}$ stems from natural dithering in the process starting times and the local planner.

The robot behavior seems not very goal directed in the first part of the experiment. This is explained in Figure 3, showing some of the plans the global planner generated by replanning. In an early stage of the conflict (Figure 3a), 


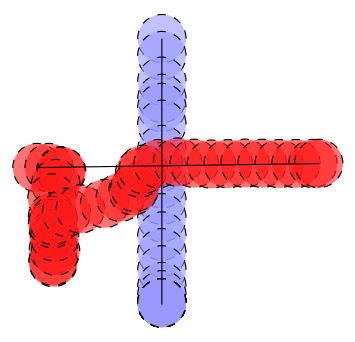

(a) One sample

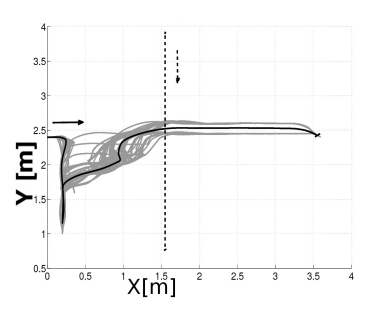

(b) Plot for 100 samples
Fig. 2: Robot behavior with cost model Static. Interferer moved from top to bottom, subject from left to right.

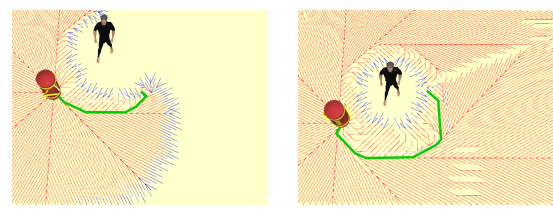

(a) Robot starts to devi- (b) Robot deviates more, (c) Robot replanned path ate from straight line becomes stalled

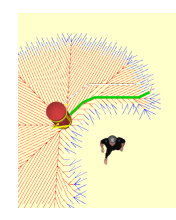

deviates by going behind the interferer
Fig. 3: Evolution of global plans during a sample trial with cost model Static. The lines on the floor are edges of the search tree (for illustration the images show breadth-first search instead of $\left.A^{*}\right)$. The thick line is the cheapest path to the goal.

the global planner finds a cheapest path moving in front of the interferer in a certain distance. As the interferer moves forward, the new navigation plans take larger detours in front of the interferer (Figure 3b). At some point the path cost is minimized by a path around the back of the interferer (Figure 3c). But until this path was considered as more appropriate, the robot had already started to execute the previous plans passing in front of the interferer. How long the robot moves in parallel to the interferer before the global planner finds a solution that passes behind the interferer depends on the individual velocities.

With respect to legibility, an observer is able to observe that the robot perceived a change of the human position as the robot reacts to it. However the nature of the robot movement obfuscates the robot intention of moving to a specific goal, reducing the legibility. Also the behavior does not seem very efficient.

\section{EXPERIMENT ON HUMAN NAVIGATION}

In order to determine a more desirable behavior for the robot, we performed an experiment, in which two people crossed each other's path. The goal was to analyze the movement characteristics, including the path taken and the velocity at each point.

We assume that a robot that moves in a (qualitatively) similar way as humans will be accepted by humans. So if a person decides to move on a curved path in a certain situation, a robot should also choose a curved path in similar situations. We use these movement characteristics as a definition of legible navigation behavior, assuming that the

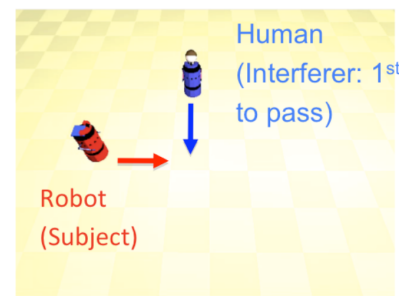

(a) In Gazebo simulator using HANP planner

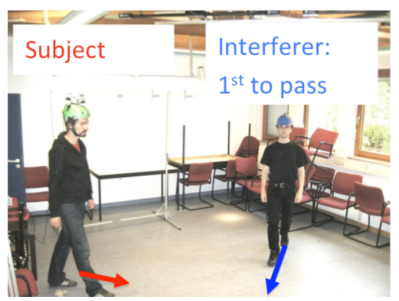

(b) In Laboratory
Fig. 4: Experimental setup.
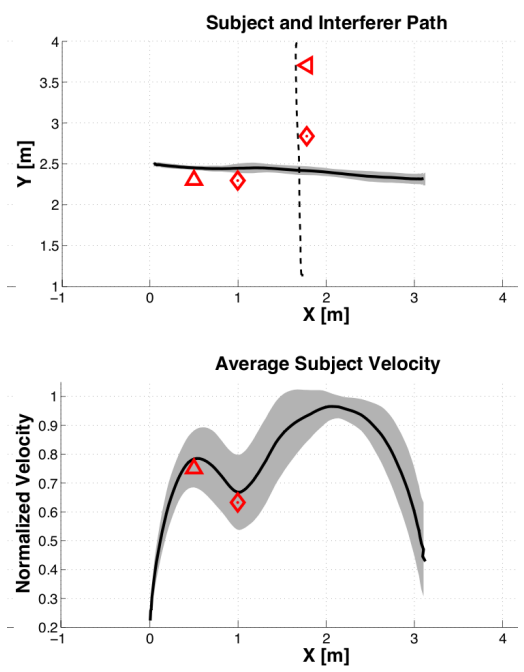

Fig. 5: The average trajectory of the subject and interferer are shown on the top. The gray area represents the standard deviation over all the subjects' paths. The red triangle indicates, on average, where the subjects started to decrease their velocity, the diamond where it started to increase again, i.e. the start and the end of the adaptation phase. On the bottom the average and standard deviation of the velocity are shown.

intentions of a robot moving in a similar manner would be clear to a human observer.

Collision avoidance among humans was analyzed in a scenario considering two persons (an interferer and a subject) with orthogonal crossing paths. The interferer was always the same person and was instructed to ignore the subject by not looking at her/him and by trying to be the first to pass. On the other hand, the subject's task was to reach a predefined goal position. In this way, we practically induced the subjects to adapt to the interferer's trajectory in order to avoid a collision. The trajectories of the subject and interferer were recorded using a motion tracking system (IS-600 Mark 2, InterSense Inc., USA) with infrared and ultrasound signals at $150 \mathrm{~Hz}$ for the subject and $20-50 \mathrm{~Hz}$ for the interferer. Both persons were wearing a helmet with a tracking sensor on it. The size of the tracked area was $4 \mathrm{~m} \times 4 \mathrm{~m}$ in the middle of a room of $38 \mathrm{~m}^{2}$ (see Figure $4 \mathrm{~b}$ ).

In the experiments participated 10 subjects ( 6 females and 4 males) between 25 and 43 years old. The subjects were 
instructed to walk to a marked point at the opposite of the room, on a direct line from their starting position. Four trials were performed.

The situation was chosen in such a way that data for interpretation of human trajectory generation and decision making could be collected. This is in contrast to other studies such as by Pacchierotti [14] who performed a user study asking subjects for feedback after having passed a robot in a real world hallway, with the intention of evaluating robot performance.

\section{A. Results}

The results showed a modification of the subjects' trajectory in terms of velocity adaptation rather than of path alteration. This velocity adaptation consisted in an initial deceleration and a subsequent acceleration within a time gap of one second, before the interferer had reached the intersection of the paths, i.e. the possible collision position (see Figure 5).

It is noticeable that the velocity profiles show two local maxima, meaning the humans did not prefer to, or were not able to, find a trajectory that has just one acceleration and deceleration phase. For robots this means that it may not be necessary to always avoid multiple local maxima in velocity for similar situations, even if avoidance would reduce jerk.

\section{B. Discussion}

For a natural trajectory, i.e. without interferences, it can be assumed that biological motion minimizes energy (the jerk) to achieve the smoothest possible trajectory [15].

This means that if we want to reach a spot ahead of us, we perform a straight-line path avoiding curved paths that would delay the reaching of our target. However, when a static obstacle is placed on our way, we are forced to adapt and replan our approach by changing our path. We could therefore expect a similar behavior when a moving obstacle, for instance a human, is interfering with our path.

Nonetheless, the experiment demonstrates, on the contrary, only a velocity adaptation. The path remains invariant over each repetition trial and subject. Therefore, the resulting trajectory for this case does not match with the expectations of minimum jerk behavior. Hence, it seems that humans perceive moving obstacles differently than static obstacles. This distinction has to be considered in a human aware navigation planner for robots accordingly.

\section{Context-dependent Cost Model}

As shown in Section III, cost model Static fails to produce legible behavior in some dynamic situations. Based on the results of the human experiment in Section IV we tried to establish an improved cost model for dynamic situations that does not have the weaknesses of cost model Static. Temporal planning was not a viable option because in order to keep the robot's behavior legible, the robot needs to remain reactive to changes. Planning with the dimension of time threatens reactivity, therefore we try to find a cost model that avoids the scaling issues of temporal planning.
This observation led us to a new cost model that does not only define a penalty on grid cells, but reduces this penalty based on the direction this grid cell is traveled to during search. This allows for social costs to exist around humans, but to reduce these social costs to zero in specific cases.

As an example, in HANP a grid cell $50 \mathrm{~cm}$ close to a moving human might have a numerical penalty of 42 during search regardless of the search context. With contextual costs, the same cell could have costs 42 for a path approaching the human from the front via this cell, meaning discomfort for the human, but costs 0 for moving in the same direction as the human via this cell. Similarly a whole path does not have the same costs regardless of what direction it is traveled in. Following a human on a path going in the same direction as the human has less costs than attempting to pass through the human in the opposite direction on the same path. This intuitive relation between path and direction of motions cannot be captured in HANP nor in any other cost model with fixed costs around humans.

To this end, we introduce the concept of path compatibility. We consider the paths of two agents to be compatible if they can be followed by both agents concurrently with velocities $v \geq 0$ at all times such that no deadlock occurs and both agents reach the end of their paths. Any two agents on compatible paths can reduce their planning efforts to modulation of forward speeds along the path, reducing their "cognitive effort". Based on this idea we may also say that the more any agent has to deviate from the intended path, or desired velocity to resolve deadlock, the less compatible two paths are.

We only estimate compatibility, as prediction of human motion is an unsolved problem. Research approaches to predict human motion for robotics are presented in [16], [17], but the approaches still have too many constraints to be generally applicable. Here we use a linear projection of the human path based on his current velocity vector.

Compatibility is just one of several factors driving path quality. We also want the robot to behave efficiently, socially compliant and legibly. As such, we do not optimize solely for compatibility, but merge compatibility as a factor in the weighted sum indicating quality of paths. Search can then still generate all paths that can be generated in HANP, but incompatible paths are less likely to be found as solutions, because more compatible paths, which have higher costs with cost model Static, will now have lower costs.

To formalize compatibility, we define a function for social costs on grid cells depending on the direction it would be reached given its predecessor cell in the path. As a consequence, the costs in each cell cannot be calculated independently of the search context.

We replace the cost function $\varsigma\left(H, w_{i}\right)$ in a grid cell for one human with a function $\varsigma^{\prime}\left(H, w_{i}, w_{i-1}\right)$ taking into account the last waypoint $w_{i-1}$ on the currently planned path $\tilde{P}=\left(w_{1}, \ldots w_{i-1}, w_{i}\right)$. The final cost function $\sigma\left(\mathscr{H}, w_{i}\right)$ considering the set of $n$ humans $\mathscr{H}$ is replaced accordingly with a cost function $\sigma^{\prime}\left(\mathscr{H}, w_{i}, w_{i-1}\right)$.

As shown in Figure 6, we use two measures to quantify 
$H$ position of human

$\hat{h d}$ heading of human

$\hat{h d}$ translated $\hat{h d}$

$\hat{h d}^{-1}$ inverse $\hat{h d}{ }^{\prime}$

$w_{i} \quad$ way point on path

$P$ closest point on human path to $w_{i}$

$d_{P} \quad$ distance of $P$ and $w_{i}$

$\hat{r d}$ potential heading of robot at $w_{i}$

$\alpha \quad$ angle between $\hat{r d}$ and $\hat{h d}^{-1}$

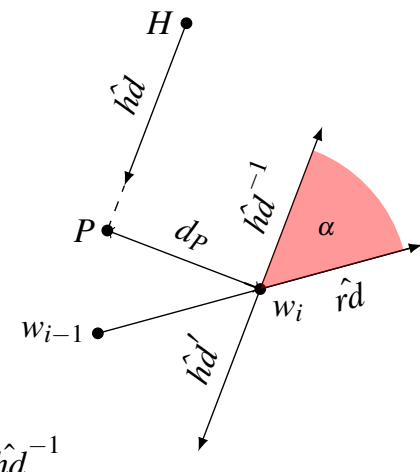

Fig. 6: Context dependent costs. The parameters for the application of social costs are distance $d_{P}$ and angle $\alpha$. If both are small, paths are incompatible, if either is large enough, paths become compatible.

path compatibility in a waypoint. Spatial compatibility is measured by the distance $d_{P}$ between the waypoint and the estimated path of the human. If this distance is large enough, the human can move along his intended path without the robot being in the way. The more distant the robot is to the path, the higher the probability that the human will not need to deviate from his intended path.

The second measure is the difference in heading angles $\alpha$, which we call directional compatibility. The angle $\alpha$ is determined by the heading of the person and the heading of the robot at the potential waypoint $w_{i}$ given by the cell of the waypoint $w_{i}$ and its previous waypoint $w_{i-1}$, as shown in Figure 6 and equation (4). We use the unit vectors $\hat{h d}^{-1}$ and $\hat{h d}$ of those directions to get the angle, normalized to the domain $[-\pi, \pi]$ :

$$
\alpha=\operatorname{norm}\left(\operatorname{acos}\left(\hat{h d}^{-1} \cdot \hat{r d}\right)\right.
$$

Using the angle $\alpha$ and distance $d_{P}$, we can define the incompatibility function $\phi$, that calculates a number $\in[0,1]$ with which we can multiply the costs of cost model Static. We define $\phi$ such that when a path segment should not conflict with a predicted human motion, social costs do not apply. $\phi$ has three parameters to tweak what motions are considered compatible.

$$
\phi\left(H, w_{i}, w_{i-1}\right)=\left\{\begin{array}{cl}
1 & , \text { if } \hat{h d} \text { undefined } \\
1 & , \text { if } d_{P} \leq d_{\text {low }} \\
0 & , \text { if } d_{P} \geq d_{h i g h} \\
0 & , \text { if } \alpha \geq \alpha_{\text {max }} \\
\frac{d_{P}-d_{\text {low }}}{d_{\text {high }}} \cdot \frac{\alpha}{\alpha_{\max }} & , \text { otherwise }
\end{array}\right.
$$

As shown in equation (5), for distance $d_{P}$, anything below $d_{\text {low }}$ is considered spatially incompatible, and anything above $d_{\text {high }}$ is considered spatially compatible. We used $d_{\text {low }}=1 \mathrm{~m}$ and $d_{\text {high }}=2 \mathrm{~m}$. For the crossing experiment, using different values did not change the behavior a lot, but in other situations it might. Similarly, angles $\alpha$ greater than $\alpha_{\text {max }}$ are considered directionally compatible.

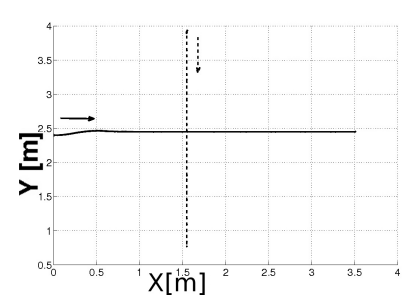

(a) Robot paths in 100 samples with cost model ContextCost

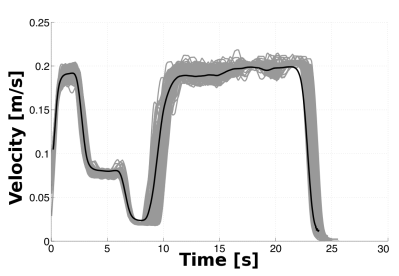

(b) Autonomous robot Velocity
Fig. 7: Robot behavior with cost model ContextCost for the crossing scenario. Interferer moved from top to bottom, subject robot from left to right.

The case of $\hat{h d}$ undefined relates to humans for which no direction could be calculated, e.g. standing humans.

For all incompatible value sets, a linear function is used to calculate degrees of incompatibility. As we have seen in the human-human experiment, at angles around $90^{\circ}$, the subject did not deviate from the shortest path. We chose $\alpha_{\max }=80^{\circ}$, and return incompatibility zero for angles greater than this.

$\varsigma_{\text {ContextCost }}^{\prime}\left(H, w_{i}, w_{i-1}\right)=\varsigma_{\text {Static }}\left(H, w_{i}\right) \cdot \phi\left(H, w_{i}, w_{i-1}\right)$

We modify cost model Static by replacing $\varsigma_{\text {Static }}$ with $\zeta_{\text {ContextCost }}^{\prime}$ as seen in equation (6).

For cost model ContextCost we also changed the original HANP neighbor predicate of Figure 1c: For all search states except those neighboring the start cell, only those neighbours were expanded whose angle from the previous grid cell was no bigger than $90^{\circ}$. So the planner could not plan to turn in points at angles greater than that. This means of the 16 directions given in Figure 1c, only 10 were used. This restriction resembles the motion primitives used in [18].

\section{A. Evaluation of Cost Models Static and ContextCost}

We performed the same evaluation experiment for cost model ContextCost as for cost model Static, and compared efficiency and behavior. We think that efficiency is one aspect of legibility. Inefficient behavior implies unnecessary actions or detours. So if an approach is very inefficient, we doubt that it would be legible. We also compare the similarity of the robot to the human behavior.

In the crossing experiment the cost model ContextCost shows the same behavior as the participants in our humanhuman experiment: The angle $\alpha$ between the heading of the person $h d$ and the movement direction of the robot $r d$ is always close to $90^{\circ}$, shown in Figure 7a. A collision between both agents was prevented by speed modulation of the autonomous robot as seen in Figure $7 \mathrm{~b}$.

Figure 8 shows the durations and distances needed to reach the goal position in the crossing scenario for the two cost models. In 100 trials, cost model ContextCost performed in a stable way despite natural dithering in the simulator. The statistics also show that efficiency was improved over the straightforward cost model Static, as the time taken with both alternative strategies dropped from around 40 seconds to 25 seconds. Without interferer, at the maximum speed of $0.2 \mathrm{~m} / \mathrm{s}$ 


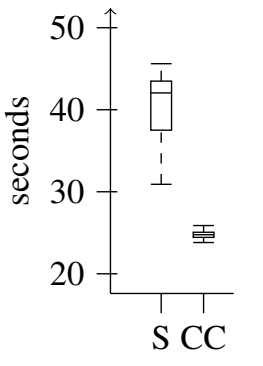

(a) Time taken

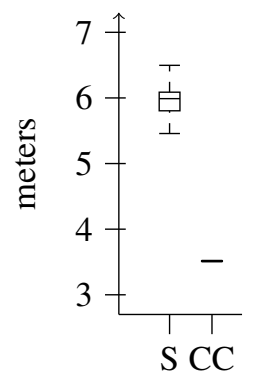

(b) Distance traveled
Fig. 8: Statistics for 100 runs for each cost model for the crossing scenario. S is for Static and CC for cost model ContextCost.The boxes indicate the range where $50 \%$ of all values occurred, the line in the box is the median, and the "whiskers" represent the minimum and maximum values, except for outliers.

allowed for the experiments, the robot would cover the same distance in 17.5 seconds.

\section{DISCUSSION}

Our original goal was to make human-aware navigation more legible in dynamic situations. We have shown that costs assuming a static world cannot directly be applied to the dynamic case without loss of legibility. By using contextdependent path compatibility, we could produce both socially acceptable and legible behavior.

The robot behavior in static cases remains unchanged from cost model Static. The impact of the new cost model in other dynamic situations remains to be investigated.

We observed that taking the shortest path to the goal for crossing scenarios appears to be the preferred human approach in experiments. Yet human-aware global planning with fixed social costs cannot generate such paths. We could reproduce the human behavior of our crossing experiment with cost model ContextCost.

The robot adapted to change in human location thanks to the local planner reducing velocity. Otherwise the robot maintained a straight line legibly demonstrating its navigation goal.

\section{CONCLUSION}

We have presented an interdisciplinary approach to make robot navigation in dynamic situations legible and socially acceptable for humans. We have shown that constant replanning with the planner HANP can lead to inefficient and illegible behavior.

To improve the robot behavior, we conducted a humanhuman experiment to find a socially plausible strategy to behave in such situations. We found human participants did not deviate from the straight path to the goal, and modulated their velocity to prevent collision. This behavior is a surprising discovery as the motion is not optimal with respect to jerk. A path planner with fixed social costs cannot find this path as a solution, yet we assume it to have a high degree of legibility.
Execution of the behavior on real robot is a necessary next step to estimate the influence of real-world factors on the approach.

We have presented a novel cost model ContextCost of using global planners with context-dependent social costs. We could show it to perform more efficiently and more human-like than the static cost model.

\section{ACKNOWLEDGMENTS}

This work was supported by the cluster of excellence Cognition for Technical Systems and by the Institute for Advanced Study of Technische Universität München, funded by the German Excellence Initiative.

\section{REFERENCES}

[1] T. Fong, I. Nourbakhsh, and K. Dautenhahn, "A survey of socially interactive robots," ROBOTICS AND AUTONOMOUS SYSTEMS, vol. 42, no. 3-4, pp. 143-166, 2003.

[2] E. A. Sisbot, L. F. Marin-Urias, R. Alami, and T. Simeon, "A human aware mobile robot motion planner," IEEE Transactions on Robotics, vol. 23, 2007.

[3] E. A. Sisbot, "Towards human-aware robot motions," Ph.D. dissertation, LAAS/CNRS, Universite Paul Sabatier, October 2008.

[4] J.-C. Latombe, Robot Motion Planning. Boston, MA: Kluwer Academic Publishers, 1991.

[5] J. P. van den Berg, M. C. Lin, and D. Manocha, "Reciprocal velocity obstacles for real-time multi-agent navigation," in ICRA, 2008.

[6] P. Trautman and A. Krause, "Unfreezing the robot: Navigation in dense, interacting crowds," in Intelligent Robots and Systems (IROS), 2010 IEEE/RSJ International Conference on, oct. 2010.

[7] S. T. Hansen, M. Svenstrup, H. J. Andersen, and T. Bak, "Adaptive human aware navigation based on motion pattern analysis," in Robot and Human Interactive Communication, 2009., Toyama, Japan, Sept.Oct. 2009.

[8] R. Gockley, J. Forlizzi, and R. Simmons, "Natural person-following behavior for social robots," in Proceedings of the ACM/IEEE international conference on Human-robot interaction, ser. HRI '07, 2007.

[9] E. Hall, The hidden dimension. Anchor Books, 1966.

[10] K. Dautenhahn, M. Walters, S. Woods, K. L. Koay, C. L. Nehaniv, E. A. Sisbot, R. Alami, and T. Siméon, "How may i serve you?: a robot companion approaching a seated person in a helping context," in ACM SIGCHI/SIGART International Conference on Human- Robot Interaction, HRI, Utah, USA, March 2006, pp. 172-179.

[11] A. Oosterhout, van T.; Visser, "A visual method for robot proxemics measurements," in Proceedings of Metrics for Human-Robot Interaction: A workshop at the Third ACM/IEEE International Conference on Human-Robot Interaction (HRI08), p.61-68., 2008.

[12] T. Kruse, A. Kirsch, E. A. Sisbot, and R. Alami, "Dynamic generation and execution of human aware navigation plans," in Proceedings of the Ninth International Conference on Autonomous Agents and Multiagent Systems (AAMAS), 2010.

[13] _ "Exploiting human cooperation in human-centered robot navigation," in IEEE International Symposium in Robot and Human Interactive Communication (Ro-Man), 2010.

[14] E. Pacchierotti, H. Christensen, and P. Jensfelt, "Evaluation of passing distance for social robots," in Robot and Human Interactive Communication, 2006. ROMAN 2006. The 15th IEEE International Symposium on, sept. 2006.

[15] G. Arechavaleta, J. Laumond, H. Hicheur, and A. Berthoz, "An optimality principle governing human walking," Robotics, IEEE Transactions on, vol. 24, no. 1, pp. 5-14, 2008.

[16] B. Ziebart, N. Ratliff, G. Gallagher, C. Mertz, K. Peterson, J. A. D. Bagnell, M. Hebert, A. Dey, and S. Srinivasa, "Planning-based prediction for pedestrians," in Proc. IROS 2009, October 2009.

[17] A. C. Gonzalez, M. Shiomi, T. Kanda, M. A. Salichs, H. Ishiguro, and N. Hagita, "Position prediction in crossing behaviors," in IEEE/RSJ International Conference on Intelligent Robots and Systems, 2010.

[18] A. Kushleyev and M. Likhachev, "Time-bounded lattice for efficient planning in dynamic environments," in Proceedings of the 2009 IEEE international conference on Robotics and Automation, ser. ICRA'09. IEEE Press, 2009. 